\title{
EINFÜHRUNG IN DIE ALLGEMEINE KONSTITUTIONS- UND VERERBUNGSPATHOLOGIE
}

\author{
EIN LEHRBUCH FÜR \\ STUDIERENDE UND ÄRZTE
}

VON

\section{DR. HERMANN WERNER SIEMENS}

MIT 80 ABBILDUNGEN UND

STAMMBÄUMEN IM TEX'

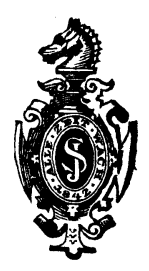

BERLIN

VERLAG VON JULIUS SPRINGER

1921 
ISBN-13:978-3-642-90412-7 e-ISBN-13:978-3-642-92269-5

DOI: $10.1007 / 978-3-642-92269-5$

Alle Rechte, insbesondere das der Übersetzung in fremde Sprachen, vorbehalten.

Copyright 1921 by Julius Springer in Berlin.

Softcover reprint of the hardcover 1st edition 1921 


\section{Vorwort.}

Umfangreiche Einführungen in die exakte Vererbungslehre haben wir genug. Ein handliches Buch aber, das aus dem ungeheuren Tatsachenmaterial dieser neuen Wissenschaft unter Vermeidung aller komplizierteren Einzelheiten nur das für den Mediziner Wichtige auswählt, und das andererseits auch auf die ärztlich besonders interessierenden Probleme der Konstitutions- und Dispositionspathologie näher eingeht, fehlte bislang; es fehlte also bislang ein Lehrbuch der Konstitutions- und Vererbungspathologie. Die vorliegende Arbeit, die sich bemühte, überall die Bedürfnisse des forschenden Arztes zu berücksichtigen, möchte diese Lücke ausfüllen.

Die Ergebnisse der mendelistischen Erblichkeitsforschung sind für jeden, der lernen will, hinter einem Stacheldraht schwer verständlicher Terminologien verborgen. Ich habe mich deshalb in meiner Darstellung jener Ausdrucksweise bedient, die sich in meinen "Grundlagen der Rassenhygiene" didaktisch bewährt und schon mehrfach Nachfolge gefunden hat.

Möge das Buch vor allem einer größeren Zahl von Kollegen die Anregung geben, zuverlässige Beobachtungen über Vererbung beim Menschen zu machen und wissenschaftlich zu bearbeiten! Denn ohne eine umfassende Vermehrung unseres exakten Beobachtungsmaterials wird es uns nicht gelingen, in die noch dunkeln Probleme der menschlichen Vererbungspathologie tiefer einzudringen.

Breslau, Ende 1920.

Herm. Siemens. 


\section{Inhaltsverzeichnis.}

\section{A. Theoretischer Teil.}

Seite

1. Die konstitutionspathologischen Grundbegriffe

Ursach e. - Die Pathologie 1. - Die Idiopathologie 1. - Die Ursachen 1. Exogene und endogene Ursachen 2. - Erbliche und nichterbliche Ursachen 2. - Idiotypische und paratypische Ursachen 3. - Phänotypus 4.

Krankheit. - Krankheit 5. - Mißbildung 5. - Anomalie 7. - Entartung 7. - Entartungszeichen 8. - Krankheit ein Relationsbegriff 8. - Krankhaftes Milieu 9. -- Symbiose 9.

Konstitution. - Konstitution und Disposition 10. - Konstitution und Idiotypus 11. - Konstitution und Phänotypus 12. - Konstitution und Konstitutionssymptome 12. - Konstitution und Anthropologie 13. - Konstitution ein Symptomenkomplex 13. - Konstitution und Krankheit 1.3. - Konstitution und endokrine Störung 14. Diathese 15. - Konstitution und Stoffwechselanomalie 15. - Definition des Konstitutionsbegriffs 15. - Idiotypische und paratypische Konstitution 16. - Die Konstitutionsanomalien: Status asthenicus 17, Status infantilis 18, Status thymico-lymphaticus 19, Status exsudativus 21, Status arthriticus 22, andere Konstitutionsanomalien 25. Die Erblichkeit der Konstitutionen 27. - Die Dispositionen der Konstitutionsanomalien 28. - Die Dispositionen der Konstitutionssymptome 28. - Disposition als spezifischer Begriff 28. - Dispositionspathologie 29. - Resistenzpathologie 29. - Aufgaben der Resistenzpathologie 30. - Dispositions- oder Resistenzsymptome 30. Funktionelle Resistenzpathologie 30. - Morphologische Resistenzpathologie 30. - Anthropologische Resistenzpathologie 31. - Methoden der Resistenzpathologie 31. - Korrelation zwischen Konstitutionssymptom und Krankheit 32. - Die Ursaehe der Korrelation 33.

Disposition. - Disposition und Krankheit 33. - Relativität des Dispositionsbegriffs 33. - Disposition und Exposition 34. - Idiotypische und paratypische Disposition 34. - Phänotypische individuelle Disposition 35. - Idiotypische individuelle Disposition 35. - Paratypische individuelle Disposition 36. -- Generelle oder Gruppendisposition 36. - Einteilung der Gruppendispositionen 36. -- Artdisposition 37. - Rassendisposition 37. - Idiotypische und paratypische Rassendisposition 37. - Anthropologische Dispositionspathologie 38. Geschlechtsdisposition 38. - Idiotypische und paratypische Geschlechtsdisposition 38. - Disposition der Konstitutionssymptome 39. Disposition der Krankheitssymptome 40. - Pathologische und physiologische Disposition 40. - Altersdisposition 41. - Modaldisposition 41. - (Berufsdisposition 41.) - (Sozialdisposition 42.) - Lokaldisposition 42. - Temporaldisposition 42. - Allgemeine und lokalisierte Disposition 43. - Zellulardispositions-Pathologie 43. - Komplexe Dispositionen 44. - Idiotypische, idiodispositionelle und paratypische Krankheiten 45 . 
Vererbung bei einem mendelnden Unterschied. - Die Entdeckung des Mendelschen Gesetzes 46. - Kreuzung bei einem mendelnden Unterschied 46. - Heterozygotie und Homozygotie 48. Verhalten der Bastarde bei Weiterzucht 49. - Rückkreuzung 49. Kreuzung bei Dominanz 50. - Dominanz und Rezessivität 50. Dominanz des Fehlens einer Eigenschaft über ihr Vorhandensein 51. - Dominanz ein Relationsbegriff 52. - Das sog. klassische Zahlenverhältnis 52.

Vererbung bei mehreren mendelnden Unterschieden. - Kreuzung bei zwei mendelnden Unterschieden 53. - Selbständigkeit der Erbeinheiten 54. - Kreuzung bei drei und mehr mendelnden Unterschieden 55. - Abhängigkeit einer Eigenschaft von mehreren Erb. anlagen (polyide Vererbung) 55. - Komplexe Eigenschaften 56. Abhängigkeit mehrerer Eigenschaften von einer Erbanlage (polyphäne Vererbung) 56. - Einfaches Mendeln 57.

3. Die zytologischen Grundlagen der Vererbungslehre . . . . . .

Die Morphologie des Idioplasmas. - Das Idioplasma 57. - Idioplasma und Zellkern 58. - Idioplasma und Chromosomen 58. - Die sog. Reifung der Geschlechtszellen 58. - Reifung der Samen- und der Eizelle 59. - Geschlechtszellenreifung und Vererbungsbiologie 61. - Zytologie und Mendelismus 62. - Geschlechtszellenreifung und Befruchtung als Ursache der Mixovariationen 62. - Die Chromosomenzahl bei der zweigeschlechtlichen Fortpflanzung 63.

Die Bestimmung des Geschlechts. - Das Problem der Geschlechtsbestimmung 63. - Die Geschlechtschromosomen 64. - Geschlechtsbestimmung bei Lygaeus 65. - Geschlechtsbestimmung bei Drosophila 65. - Geschlechtsbestimmung bei Abraxas 67. - Geschlechtsbestimmung beim Menschen 68. - Geschlecht und Idiotypus 68.

Die Sexualproportion . . . . . . . . . . . . . . 69

Die Kontinuität des Idioplasmas. . . . . . . . . . . . . . . 70

4. Die theoretischen Grundlagen der Vererbungslehre. . . . . . . 71

Das Mendelsohe Gesetz. - Paarigkeit der Erbanlage 71. - Der biologische Vererbungsbegriff 72. - Bestätigung von Weismanns Lehren 73. - Ewigkeit des Idioplasmas 73. - Homozygotie und Heterozygotie 74. - Unvollständige Dominanz 74. - Unregelmäßige Dominanz 74. - Epistase und Hypostase 74. - Idiotypische Verschiedenheit gleich aussehender Individuen 75. - Idiotypische Gleichheit verschieden aussehender Individuen 76. - Eineiige Zwillinge 76. Idiophorie 76. - Das Mendelsche Gesetz 77.

Der Begriff der Erblichkeit. - Angeboren und idiotypisch 77. Erworben und paratypisch 78. - Erblichkeit von Zuständen und Vorgängen 78. - Erblichkeit von Eigenschaften und Reaktionsweisen 79. - Erblichkeit von Krankheitsdispositionen 80. - Erblichkeit von Merkmalen 80. - Verschiedene Reaktionsweisen idiotypisch gleicher Individuen 81. - Erblichkeitsbegriff ein Relationsbegriff 81. - Erblichkeit und Variabilität 84. - Erblichkeit von Eigenschaften, Krankheiten usw. 84. - Erblichkeit ein absoluter Begriff 85 .

Die sog. Vererbung erworbener Eigenschaften. - I. Der Ausdruck 85. - II. Die deduktiven Beweise 86. - Die Paraphorie 87. - Die Idiokinese 88. - Die Anpassung 89. - III. Die induktiven Beweise 89. - Die Beweise für die Nichtvererbbarkeit erworbener Eigenschaften 90. - Die Versuche mit reinen Erbstämmen 91. Der Mendelismus 92. - Die neuauftretenden Idiovariationen 93. IV. Die gefühlsmäßigen Gründe 93. - V. Die Bedeutung des Lamarckismus 94. - Bedeutung des Lamarckismus für Züchter, Pädagogen, Politiker, Ärzte usw. 95. - Der Lamarckismus in der Medizin 96. 
5. Die vererbungsbiologischen Grundbegriffe

Trennung von Erblichem und Nichterblichem 96. -- Keimplasma und Soma 97. - Idiotypus und Phänotypus 97. - Idiotypus und Paratypus 97. - Das Wesen des Idiotypus 98. - Erbformeln 98. - Idiokinese und Parakinese 99. - Idiophorie und Paraphorie 99. - Paratypische Eigenschaften 100. - Idiotypische Eigenschaften 100. Eltern und Kinder 100. - Übersicht 101.

\section{B. Praktischer Teil.}

6. Sammlung und Aufzeichnung vererbungswissenschaftlichen Materials beim Menschen 103

Genealogie 103. - A-Tafel und D-Tafel 103. - Stammbaum 103.

Stammbaum und Fortpflanzung 104. - Stammbaum der Familie Siemens 106. - A-Tafel 106. - Ahnenverlust 108. - Häufigkeit des Ahnenverlustes 110. - Inzucht 111. - Angebliche Schädlichkeit der Inzucht 111. - Vereinigung von A-Tafel und D-Tafel 112. Familienforschung und Vererbungspathologie 112. - Numerierung der A-Tafel 113. - Anlage und Numerierung der D-Tafel 114. Erbbiographische Personalbogen 115. - Wert der Familienforschung für die Vererbungsbiologie 115. - Vereinigung von A- und D-Tafel in der Vererbungspathologie 116.

7. Beurteilung vererbungswissenschaftlichen Materials beim Menschen . . . . . . . . . . . . . . . . . . . . . . . . . . .

Dominante Vererbung. - Zahlenverhältnisse 117. - Homozygotie 120. - Selektionswirkungen 121. - Nachweis der dominanten Erblichkeit 122. - Ubersicht 122.

Unregelmäßig dominante Vererbung. - Uberspringen 123. - Fehlerhafte Anamnese 123. - Unregelmäßigkeiten der Manifestation 125. - Manifestationsschwankungen infolge Paravariabilität 127. Manifestationsschwankungen infolge Mixovariabilität 127. - Später oder wechselnder Manifestationstermin 128. - Manifestationsschwund 129. - Neuauftreten dominanter Krankheiten 129. - Abweichende Zahlenverhältnisse 130. - Größere Sterblichkeit der Behafteten 131. - Die Familie Nougaret 132. - Direkte, nicht einfach dominante Vererbung 133.

Rezessive Vererbung. - Zahlenverhältnisse 135. - D-Tafeln und A-Tafeln bei rezessiven Krankheiten 137. - Gehäufte Blutsverwandtschaft der Eltern 140. - Übersicht 142.

Unregelmäßig rezessive Vererbung. - Fehlerhafte Anamnese 143. Manifestationsschwankungen 143. - Später oder wechselnder Manifestationstermin 143. - Manifestationsschwund 144. - Größere Sterblichkeit der Behafteten 144. - Idiotypische und paratypische Formen der gleichen Krankheit 144. - Verschiedene idiotypische Formen der gleichen Krankheit 145. - Fehlerhafte statistische Methodik 145.

Dominant-geschlechtsgebundene Vererbung. - Zahlenverhältnisse 146. - D-Tafeln bei dominant-geschlechtsgebundenen Krankheiten 148. - UUbersicht 148.

Rezessiv-geschlechtsgebundene Vererbung. - Zahlenverhältnisse 149. - D-Tafeln bei rezessiv-geschlechtsgebundenen Krankheiten 151. - Die Hämophilie 153. - Geschlechtsgebundene und geschlechtsbegrenzte Vererbung 153. - Übersicht 154.

Dominant-geschlechtsbegrenzte Vererbung. - Zahlverhältnisse 156. - D-Tafeln bei dominant-geschlechtsbegrenzten Krankheiten 157.

Rezessiv-geschlechtsbegrenzte Vererbung . . . . . . . . . 158

Vererbung durch den Mannesstamm . . . . . . . . . . . . . . 159 
Seite

Vererbung durch den Weibesstamm . . . . . . . . . . . . . 160

Polyide Vererbung . . . . . . . . . . . . . . . . . . . . . . . . 160

Polyphäne Vererbung. . . . . . . . . . . . . . . . . . . . . . 161

Heterophäne Vererbung . . . . . . . . . . . . . . . . . . . . . . 161

Verschiedene Vererbungsmodi bei einer Krankheit. . . . . 165

Richtlinien für das Sammeln von vererbungswissenschaftlichem Material . . . . . . . . . . . . . . . . 166

Das Auszählen der Mendelschen Proportionen .

8. Diagnostik erblicher Krankheiten . . . . . . . . . . . . . . 171

Fehlen äußerer Ursachen 171. - Familiäres Auftreten 173. - Mendelsche Zahlenverhältnisse 174. - Blutsverwandtschaft der Eltern 174. - Krankheitsverlauf 175. - Klinisches Krankheitsbild 175.

9. Ätiologie erblicher Krankheiten . . . . . . . . . . . . . . .

Idiokinese. - Die Ursache der erblichen Krankheiten 175. - Die Ursache der erblichen Krankheitsanlagen 175. - Idiokinese bei Pflanzen und Tieren 176. - Idiokinese beim Menschen 177. Häufigkeit der Idiovariationen 180. - Idiokinese und Selektion 180.

Selektion. - Fekundative Selektion 181. - Eliminatorische und elektive Selektion 181. - Degeneration 184. - (Anteposition 184.) - Gesundheitspolitik 185. - Kontraselektion 186. - Kontraselektion zwischen den sozialen Ständen 187. - Kontraselektion zwischen den Berufsgruppen 189. — Ausdehnung der Selektionswirkung 190. - Unwiderruflichkeit der Selektionswirkung 190. - Die Proletarisierung unseres Nachwuchses 190. - Kontraselektion zwischen den Völkern 191. - Kontraselektion zwischen den großen Rassen 191.

10. 'Therapie erblicher Krankheiten . . . . . . . . . . . . . . . . 192 Therapie. -- Symptomatische Therapie 192. - Kausale Therapie 192.

1. Parakinese. - Fortpflanzungshygiene 193.

2. Idiokinese. - Homologe Idiovariationen 194. - Idiokinese und Umwelt 196. - Ausschaltung der Idiokinese 197.

3. Selektion. - Verhinderung von Verwandtenehen 200. - Erbhygienische Eheberatung 200. - Rassenhygiene 201. - Eliminatorische Rassenhygiene 201. - Elektive Rassenhygiene 201.

Rassenhygienische Geburtenpolitik. - Die Geburtenpolitik 202. - Rassenhygienische Finanzpolitik 204. - Rassenhygienische Siedlungspolitik 204. - Rassenhygienische Ethik 204. - Rassenhygiene als Lehrfach 205.

\section{Anhang.}

Úberblick über die spezielle Vererbungspathologie . . . . . . 206

Überblick über die vererbungsbiologische Terminologie. . . . 213

Ưberblick über die vererbungspathologische Literatur . . . . 216

Namenregister . . . . . . . . . . . . . . . . . 220

Sachregister . . . . . . . . . . . . . . . . . . . . . . . . 222 